\title{
Panretinal photocoagulation versus intravitreal bevacizumab for proliferative diabetic retinopathy treatment
}

\begin{abstract}
Purpose: It is to determine the visual acuity outcomes at 1 year in eyes with proliferative diabetic retinopathy using pan retinal photocoagulation compared to intravitreal Bevacizumab $1.25 \mathrm{mg}$ instead of Ranbizumab to lower the cost burden.

Methods: 30 eyes of 30 patients diagnosed with proliferative diabetic retinopathy and have randomized for either pan retinal laser photocoagulation or intravitreal Bevacizumab every 4 weeks based on retreatment protocol both groups can receive intravitreal bevacizumab or focal/grid laser for diabetic macular edema.

Main outcome and measures: A complete exam including best corrected visual acuity changes within 52 weeks, improvement in diabetic retinopathy, central retinal thickness and cost analysis.

Results: Best corrected visual acuity changed from the baseline 0.60 LogMAR in the of Bevacizumab group and 0.50 LogMAR in the PRP group, to 0.40 LogMAR in the Bevacizumab group and 0.50 LogMAR in the PRP group at week $52(\mathrm{P}=0.14)$, where central retinal thickness changes at 52 weeks from baseline -48 (-62 to -32$)$ in the Bevacizumab group and $-16(-21$ to -8$)$ in the PRP group $(\mathrm{p}<0.001)$ however proportion of eyes without active or regressed neovascularization at 52 weeks are statistically not significant between two groups $(\mathrm{p}=0.43)$.

Conclusion: Intravitreal Bevacizumab is non-inferior to pan retinal photocoagulation interim of visual acuity outcome within 52 weeks in case of proliferative diabetic retinopathy and may be more cost effective in selected cases of proliferative diabetic retinopathy with diabetic macular edema than both panretinal photocoagulation and Ranibizumab but a long term and larger studies are needed.
\end{abstract}

Volume 7 Issue I - 2017

\author{
Ameen Marashi,' Issa Abukhalaf, ${ }^{2}$ Rasha \\ Alfaraji, ${ }^{3}$ Yaman Choman, ${ }^{4}$ Alaa salahieh ${ }^{5}$ \\ 'Retina specialist Marashi eye clinic, Syria \\ ${ }^{2}$ Ophthalmology Specialist, AlBashir Hospital, Jordan \\ ${ }^{3}$ Ophthalmologist at Jordanian MOH, AlBashir Hospital, Jordan \\ ${ }^{4}$ Yamman Shuman Retina specialist at advanced ocular center \\ alkalmat hospital, Syria \\ ${ }^{5}$ AlaaSalahia Resident of opthalamology at Marashi Eye Clinic, \\ Syria
}

Correspondence: Ameen Marashi, Retina specialist at Marashi eye clinic, Aleppo, Syria,

Email ameenmarashi@hotmail.com,www.amretina.tk

Received: June 23, 2017 | Published: June 30, 2017
Abbreviations: PDR, proliferative diabetic retinopathy; VEGF, vascular endothelium growth factor; DR, diabetic retinopathy; DME, diabetic macular edema; CSME, clinically significant macular edema; BCVA, best-corrected visual acuity; CRT, central retinal thickness; OCT, ocular computer tomography; PRP, pan retinal photocoagulation

\section{Introduction}

Proliferative diabetic retinopathy (PDR) is the main cause of late onset visual loss of diabetic retinopathy and without treatment $50 \%$ of patients with high-risk proliferative diabetic retinopathy will loss vision within 5 years, ${ }^{1}$ Pan retinal laser photocoagulation has been treatment of choice for PDR in the past decades, but PRP can cause peripheral visual field loss while intravitreal vascular endothelial growth factor (VEGF) blockade agents has proven it safety and efficacy for treatment of central diabetic macular edema, it has been noted that those agents has reduced PDR worsening and maybe improvement. Protocol S by DRCR.net ${ }^{2}$ has shown that receive Ranibizumab as anti-vascular endothelial growth factor (anti-VEGF) therapy with deferred panretinal photocoagulation are non-inferior to those in eyes that receive standard prompt PRP therapy, however with some visual functional benefits and less complications with Ranibizumab arm.
Applying Protocol S in real world scenario may add cost burden to the patient as patients need about 7 injections per year which will cost the patient about 7000 US dollars a year as minimum. The primary objective of this protocol is to determine the visual acuity outcomes at 1 year in eyes with proliferative diabetic retinopathy (PDR) using Bevacizumab $1.25 \mathrm{mg}$ instead of Ranibizumab to lower the cost burden.

\section{Methods}

This is a randomized multicenter and open label double arm interventional study that adhered to the declaration of Helsinki, the study was registered at clinical trials.gov (NCT02705274), Study received approval by multiple institutional review board, and signed consent was obtained by patients after explaining the possible complications.

\section{Study participants \\ Inclusion Criteria:}

i. Age $>=18$ years

ii. Diagnosis of diabetes mellitus (type 1 or type 2 ) 
iii. Presence of PDR which the investigator intends to manage with PRP alone but for which PRP can be deferred for at least 4 weeks in the setting of intravitreal Bevacizumab, in the investigator's judgment.

iv. Best corrected Snellen equivalent 20/320 or higher on the day of randomization.

v. Media clarity, pupillary dilation, and study participant cooperation sufficient to administer PRP and obtain adequate fundus photographs and OCT.

\section{Exclusion Criteria:}

i. Significant renal disease, defined as a history of chronic renal failure requiring dialysis or kidney transplant.

ii. Myocardial infarction, other acute cardiac event requiring hospitalization, stroke, transient ischemic attack, or treatment for acute congestive heart failure within 4 months prior to randomization

iii. Systemic anti-VEGF or pro-VEGF treatment within 4 months prior to randomization.

iv. For women of child-bearing potential: pregnant or lactating or intending to become pregnant within the next 3 years.

v. Tractional retinal detachment involving the macula. -- A tractional retinal detachment is not an exclusion if it is outside of the posterior pole (not threatening the macula) and in the investigator's judgment, is not a contraindication to intravitreal Bevacizumab treatment and also does not preclude deferring PRP for at least 4 weeks in the setting of intravitreal Bevacizumab

vi. Macular edema is present that is considered to be related to ocular surgery such as cataract extraction or clinical exam and/or OCT suggest that vitreoretinal interface abnormalities disease (e.g., a taut posterior hyaloid or epiretinal membrane) is the primary cause of any macular edema.

vii. An ocular condition is present (other than diabetic retinopathy) that, in the opinion of the investigator, might alter visual acuity during the course of the study (e.g., retinal vein or artery occlusion, uveitis or other ocular inflammatory disease, neovascular glaucoma, Substantial cataract that, in the opinion of the investigator, is likely to be decreasing visual acuity by 3 lines or more (i.e., cataract would be reducing acuity to $20 / 40$ or worse if eye were otherwise normal).

viii. History of intravitreal anti-VEGF treatment at any time in the past 2 months.

ix. History of corticosteroid treatment (intravitreal) at any time in the past 4 months.

x. History of major ocular surgery (including vitrectomy, cataract extraction, scleral buckle, any intraocular surgery, etc.) within prior 4 months or anticipated within the next 6 months following randomization.

xi. Exam evidence of severe external ocular infection, including conjunctivitis, chalazion, or substantial blepharitis

xii. Uncontrolled glaucoma (in investigator's judgment).

xiii. Aphakia

\section{Treatment protocol}

One or both eyes can be included in this study, where either intravitreal Bevacizumab $1.25 \mathrm{mg}$ or PRP is assigned for the patient however PRP can be introduced whenever treatment failure in bevacizumab arm and bevacizumab can be used to address DME as needed. In Bevacizumab (1.25 mg) group study eyes received three injections every 4 weeks and PDR is re assessed at week 1820 , retreatment was considered if needed based on investigator assessment of proliferation status (neovascularization on fundus exam or retinal images) repeat injection in case of PDR worsening every 4 weeks and then stopped whenever a stable PDR is achieved for the last 2 injections, PRP can be used in bevacizumab arm in case of faulty; In PRP arm, laser is done in 1-3 secessions (1200-1600 burns), and repeated in case of PDR worsening, however vitrectomy can be done in case of non-clearing vitreous hemorrhage or tractional retinal detachment in both groups based on investigator discretion. Diabetic macular edema was defined as central or non-central CSME based on OCT as retinal thickening and was treated in both groups in the same manner, in non-central focal/modified grid using $532 \mathrm{~nm}$ continuous wave modes was used for treatment. In central DME monthly intravitreal injection of $1.25 \mathrm{mg}$ Bevacizumab used for treatment and retreatment of DME was based on presence of retinal thickening on OCT.

\section{Intervention}

Bevacizumab comes in a single used vial $100 \mathrm{mg} / 4 \mathrm{ml}$, we injected $0,05 \mathrm{ml} / 1.25 \mathrm{mg}$ of Bevacizumab using $1 \mathrm{ml}$ syringes with Luer-Lok tip, clear polycarbonate barrel after withdrawal from vial under sterile conditions hood after storing it in the refrigerator in 4 degrees Celsius. Administration of injections was done under sterile condition, where injection site was prepared by disinfecting the skin using povidone iodine $10 \%$ and the conjunctiva using povidone iodine $4 \%$ after installing topical anesthesia, the injection is carried out after placing sterile drape and lid speculum isolating eye lashes in the superior temporal quadrant, injection site was measured with calipers $4 \mathrm{~mm}$ from the limbus in phakic patients and $3.5 \mathrm{~mm}$ in pseudophakic patients, 30 gauge half inch needle is used to inject $0,05 \mathrm{ml} / 1.25 \mathrm{mg}$ of Bevacizumab then cotton tip applicator is placed over the injection site to prevent reflux of fluid, topical and systemic antibiotics were used pre and post injection. Where in PRP arm $532 \mathrm{~nm}$ diode laser was used to perform PRP in one or more sessions 1200-1600 burns using conventional laser

\section{Follow up and outcome measures}

All patients had BCVA (best corrected visual acuity) at baseline, and every 4 weeks using snellen chart then converted to LogMAR to monitor visual acuity improvement for one year where SD-OCT (OTI:OCT/SLO Heidelberg Engineering) was obtained at baseline and week 12 and then every 8 weeks to evaluate presence of DME, along with digital fundus colored photograph was obtained at baseline and week 12 and then evert 8 weeks to evaluate retinal structure changes for one year, where Intraocular pressure and cataract along with systemic events were recorded monthly.

\section{Statistical methods}

Clinical data obtained were analyzed using SPSS V.20.0 Paired student $t$ Test Calculator for 2 Independent Means was used to analyze the difference between the mean 12 weeks outcome and mean baseline values for visual acuity and CMT. 


\section{Results}

This study has randomized 30 eyes for 30 patients with PDR for either intravitreal Bevacizumab or PRP between February and April 2016, as for the Bevacizumab group number of eyes were enrolled 15, the median age was 52 (46-59), $20 \%$ of them were men and $80 \%$ were women, $60 \%$ were Arab, $40 \%$ were Caucasian and $80 \%$ had DME at the baseline where in PRP group number of eyes were enrolled 15 the median age was 53 (48-61), 50\% of them were men, $100 \%$ were Arab and 20\% had DME at the baseline (Table 1). The mean BCVA at the baseline in Bevacizumab group was $0.60 \pm 0.2 \operatorname{LogMAR}(20 / 80)$ and $0.50 \pm 0.3 \operatorname{LogMAR}(20 / 60)$ in the PRP group In the Bevacizumab group 12 patients with DME where presented and required median of $9(8-11)$ injections within 52 weeks to treat both DME and PDR, where the 3 patients that didn't present with DME at the baseline required median 7 (6-9) injection within 52 weeks to treat PDR. In the PRP group, laser photocoagulation were performed at the baseline and 10 patients $(67 \%)$ required retreatment with laser within approximately 6 months, however the 3 patients had DME at the baseline where treated with focal laser as the DME was non central CSME.

According to Table 2 LogMAR changed from the baseline 0.60 LogMAR (20/80) in the of Bevacizumab group and 0.50 LogMAR (20/60) in the PRP group, to $0.40 \operatorname{LogMAR}(20 / 50)$ in the
Bevacizumab group and 0.50 LogMAR (20/60) in the PRP group at week $52(\mathrm{P}=0.14)$ (Figure 1). At 52 weeks intravitreal Bevacizumab was non-inferior to PRP interim of visual acuity, and there is no significant change in visual acuity, BCVA was converted from Snellen to LogMAR for statistical analysis. As shown in Table 2 central retinal thickness changes at 52 weeks from baseline -48 (-62 to -32) in the Bevacizumab group and $-16(-21$ to -8$)$ in the PRP group $(\mathrm{p}<0.001)$ however the probability of developing DME was higher in the PRP group, due to the small group of patients, and the randomization" where DME patients exceeds PRP patients"; we can't have a firm conclusion. The improvement of diabetic retinopathy at 52 weeks was 11 eyes without PDR, 3 eyes with regressed neovascularization, 2 eyes with active neovascularization and 9 eyes improved two steps in DR severity in Bevacizumab group (Figure 2) where 4 eyes without PDR, 9 eyes with regressed neovascularization, and 1 eye with active neovascularization in PRP group $(\mathrm{p}=0.43)$ where the two steps improvement in DR severity was non assessable in the PRP group (Figure 3). No systemic nor ophthalmic events where noted in both arms of the study except of one patient in the Bevacizumab groups developed hyphema and vitreous hemorrhage the next day after injection and was treated mydriatic and topical steroids which hemorrhage has resolved without any ocular or retinal damage then scheduled for PRP.

Table I Characteristics of patients at presentation

\begin{tabular}{|c|c|c|}
\hline & Bevacizumab group & PRP group \\
\hline No & 15 & 15 \\
\hline Age (mean) & $52(46-59)$ & $53(48-6 I)$ \\
\hline $\begin{array}{l}\text { Sex N (\%) } \\
\text { Men } \\
\text { Female }\end{array}$ & $\begin{array}{l}\text { Male } 3(20 \%) \\
\text { Female I2 (80\%) }\end{array}$ & $\begin{array}{l}\text { Male } 4(27 \%) \\
\text { Female II (73\%) }\end{array}$ \\
\hline $\begin{array}{l}\text { Eye N (\%) } \\
\text { Right } \\
\text { Left }\end{array}$ & $\begin{array}{l}\text { Right } 60 \% \\
\text { Left } 40 \%\end{array}$ & $\begin{array}{l}\text { Right } 50 \% \\
\text { Left } 50 \%\end{array}$ \\
\hline $\begin{array}{l}\text { Race N (\%) } \\
\text { Arab } \\
\text { Caucasian }\end{array}$ & $\begin{array}{l}\text { Arab } 9(60 \%) \\
\text { Caucasian } 6(40 \%)\end{array}$ & Arab I5 (100\%) \\
\hline $\begin{array}{l}\text { Lens: N (\%) } \\
\text { Phakic } \\
\text { Pseudophakic }\end{array}$ & $\begin{array}{l}\text { Phakic II (73\%) } \\
\text { Pseudophakic } 4 \text { (27\%) }\end{array}$ & $\begin{array}{l}\text { Phakic I4 (94\%) } \\
\text { Pseudophakic I (6\%) }\end{array}$ \\
\hline DME N (\%) & $12(80 \%)$ & $3(20 \%)$ \\
\hline $\begin{array}{l}\text { Other Systemic } \\
\text { Hypertension } \\
\text { Cardiovascular }\end{array}$ & Hypertension 6 (40\%) & Hypertension $3(20 \%)$ \\
\hline
\end{tabular}

Table2 Mean changes of BCVA (best corrected visual acuity) in LogMAR units, CRT (Central retinal thickness), and diabetic retinopathy (DR) changes from baseline and after 52 weeks

\begin{tabular}{llll}
\hline Outcomes & Bevacizumab & PRP & P Value \\
\hline Number of patients & 15 & 15 & 0.5 \\
Mean BCVA at 52 weeks in LogMAR & 0.4 & 0.5 & 0.14 \\
Mean BCVA at baseline in LogMAR & 0.6 & $246(24 I$ to 253$)$ & $258(256$ to 26 I) \\
Mean CRT at 52 weeks & $-48(-62$ to -32$)$ & $-16(-21$ to -8$)$ & $<.00$ I \\
Changes of CRT from baseline & & & 0.43 \\
Diabetic retinopathy changes at 52 weeks & 11 & 4 & 9 \\
No of Eyes without PDR at 52 weeks & 3 & I \\
No of Eyes with regressed neovascularization & 2 & N/A \\
No of Eyes with active neovascularization & 9 & & \\
No of Eyes improved 2 steps in DR severity & & & \\
\hline
\end{tabular}




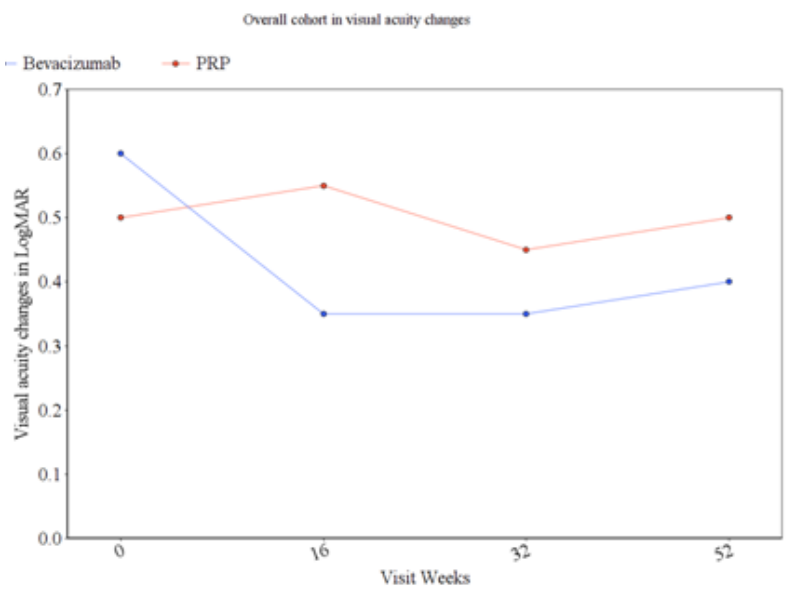

Figure I 95\% Confidence interval- BCVA, LogMAR units according to time assessment.

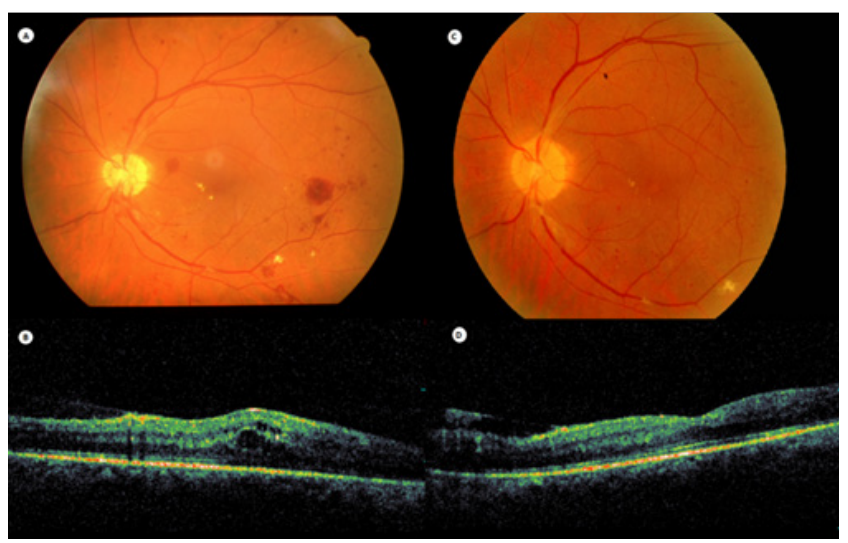

Figure $2 \mathrm{~A}$ and $\mathrm{B}$ patient with PDR and DME at the baseline BCVA is 20/40; $C$ and D the same patient after 52 weeks shows complete regression of neovascularization and DME improvement after receiving 9 injections of intravitreal Bevacizumab, BCVA 20/20.

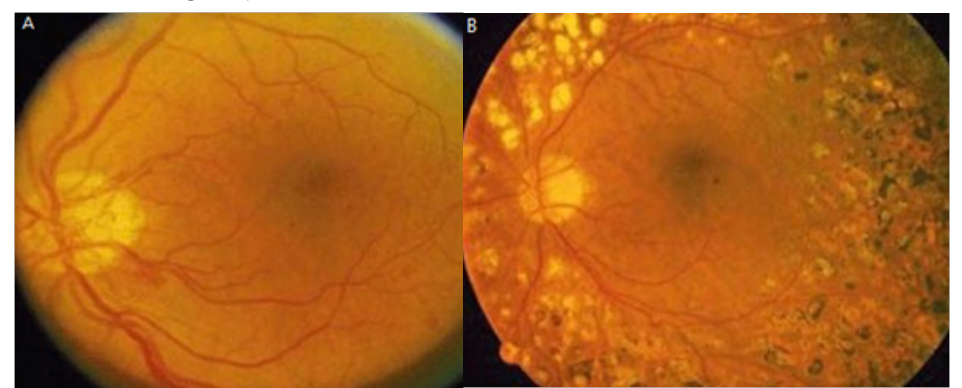

Figure 3 A shows a patient with PDR, baseline BCVA is 20/63; B shows same patient after 52 weeks Panretinal photocoagulation (PRP), BCVA $20 / 32$.

\section{Discussion}

VEGF is up regulated as a response to relative ischemic state in diabetic retinopathy and can induce the process of angiogenesis, which lead to progression of PDR; ${ }^{3}$ clinical trials have reported the effect of VEGF blockade agents on slowing the progress of diabetic retinopathy and reduction of its complications but the VEGF blockade agents have short-term effect. DRCR.net have evaluated the effect of VEGF blockade agents in treatment of PDR and its complications, Protocol $\mathrm{S}$ has evaluated the non-inferiority of visual acuity for intravitreous ranibizumab vs. PRP for PDR and they concluded that intravitreous ranibizumab is non-inferior to PRP with less PDR complications in the ranibizumab arm, however patients presented with DME and PDR have gained more visual acuity in the Ranibizumab arm. ${ }^{2}$ This randomized clinical trial has shown, that intravitreal bevacizumab is non inferior interim of visual acuity changes than PRP in the settings of PDR in one year, as there is no statistical deference between two groups and thus meet the primary outcome in this study. However, patients that presented with central diabetic macular edema and PDR had better visual outcome when treated with bevacizumab in contrast to the patients who were treated with PRP, where in patients who did not show any signs of DME at baseline, both groups had no statistical deference interim visual acuity however DME development was more common in the PRP group.

As secondary outcome measures, PRP may be more cost effective in the absence of DME at the baseline but in the presence of DME, intravitreal Bevacizumab is more cost effective interim of life quality as intravitreal Bevacizumab may improve both vision ${ }^{4}$ and retinal anatomy and reduce the need of vitrectomy, where the cost of laser 
treatment is about 200 USD per session; Bevacizumab may cost 60 USD per injection and when compare it to intravitreal Ranibizumab costs about 1100 USD per injection, Bevacizumab seems to bean alternative interim of cost to Ranibizumab for PDR but intravitreal Ranibizumab may have better PDR improvement statically (about $20 \%$ ) in the settings of DME as shown in the secondary analysis by DRCR.net ${ }^{5}$ but with higher price tag. In the Bevacizumab group, diabetic retinopathy has improved two steps, in contrast to PRP group which diabetic retinopathy improvement was not assessable but it is important to take into account that PRP group needed less treatment to stabilize the diabetic retinopathy in contrast to bevacizumab group which may need repeatable injections to stabilize the diabetic retinopathy, where active neovascularization and vitreous hemorrhage may be recur after stopping injection.

Central macular thickness was markedly reduced in the Bevacizumab group in cases of DME at the baseline and the risk of DME development was lower in Bevacizumab group in contrast to PRP group, however changes in central retinal thickness among eyes without DME at the baseline was clinically insignificant. Taking into account the results and analysis of Protocol T by DRCR.net, ${ }^{6,7}$ intravitreal Bevacizumab maybe more effective in cases of good vision at the baseline and central retinal thickness 400 microns and less, which meets the results of this study but this should be viewed with caution to avoid bias due to small amount of recruitment in this study. PDR complications such as vitreous hemorrhage and tractional retinal detachment along with the need of vitrectomy were not included in this study due to the small number of recruitment and short term follow-up. Applying the results of this study in every day practice, PDR patients should be classified as without macular edema or with macular edema, where the former PRP may be more cost effective and the latter intravitreal Bevacizumab is more cost effective because Bevacizumab is treating both DME and PDR with better results, however PRP can be differed in the presence of DME with PDR in cases of treatment failure or it is hard to follow up the patient.

Limitation of this study are the following:

1. Small amount of recruitments

2. Short time follows up.

3. Unknown deference in visual filed sensitivity between two groups

4. Unknown the long-term visual outcome between two groups.

5. Unknown the long-term systemic and ocular safety for using intravitreal bevacizumab as solo treatment for PDR.

\section{Conclusion}

PDR treatment with intravitreal Bevacizumab was not inferior to PRP at 52 weeks follow-up, despite the limitation of this study Bevacizumab may offer good alternative to PRP for PDR patients in the settings of DME at baseline and maybe more cost effective than intravitreal Ranibizumab in selected cases, however a larger studies with long-term follow-up are warranted.

\section{Acknowledgments}

None.

\section{Conflicts of interest}

The authors declare no conflicts of interest.

\section{References}

1. Diabetic retinopathy study research group. photocoagulation treatmen of proliferative diabetic retinopathy: clinical application of diabetic retinopathy study (drs) findings, DRS report number 8. Ophthalmology. 1981;88(7):583-600.

2. A randomized clinical trial writing committee for the diabetic retinopathy clinical research network JAMA. Panretinal Photocoagulation vs Intravitreous Ranibizumab for Proliferative Diabetic Retinopathy. 2015;314(20):2137-2146

3. Taylor E, Dobree JH. Proliferative diabetic retinopathy. Site and size of initial lesions. Br J Ophthalmol.1970;54(1):11-18

4. Ross EL, Hutton DW, Stein JD, et al. Cost-effectiveness of aflibercept, bevacizumab, and ranibizumab for diabetic macular edema treatmentanalysis from the diabetic retinopathy clinical research network comparative effectiveness trial. JAMA Ophthalmol. 2016;134(8):888896.

5. Bressler SB, Liu D, Glassman AR, et al. Change in diabetic retinopathy through 2 yearssecondary analysis of a randomized clinical trial comparing aflibercept, bevacizumab, and ranibizumab. JAMA Ophthalmol. 2017;135(6):558-568.

6. Jampol LM, Glassman AR, Bressler NM, et al. Anti-vascular endothelial growth factor comparative effectiveness trial for diabetic macular edema additional efficacy post hoc analyses of a randomized clinical Trial. JAMA Ophthalmol. 2016;134(12):1429-1434.

7. Wells JA, Glassman AR, Ayala AR, et al. Aflibercept, bevacizumab, or ranibizumab for diabetic macular edema. $N$ Engl $J$ Med. 2015;372(13):1193-1203 